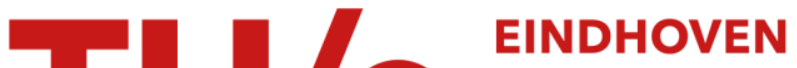 \\ UNIVERSITY OF \\ TECHNOLOGY
}

\section{Multivariable control design for fixed direction disturbances}

\section{Citation for published version (APA):}

Boerlage, M. L. G., Jager, de, A. G., \& Steinbuch, M. (2007). Multivariable control design for fixed direction disturbances. In Proceedings of the 2007 American Control Conference (ACC 2007) 9-13 July 2007, New York, New York, USA (pp. 4649-4654). Institute of Electrical and Electronics Engineers.

https://doi.org/10.1109/ACC.2007.4283017

DOI:

10.1109/ACC. 2007.4283017

Document status and date:

Published: 01/01/2007

\section{Document Version:}

Publisher's PDF, also known as Version of Record (includes final page, issue and volume numbers)

\section{Please check the document version of this publication:}

- A submitted manuscript is the version of the article upon submission and before peer-review. There can be important differences between the submitted version and the official published version of record. People interested in the research are advised to contact the author for the final version of the publication, or visit the $\mathrm{DOI}$ to the publisher's website.

- The final author version and the galley proof are versions of the publication after peer review.

- The final published version features the final layout of the paper including the volume, issue and page numbers.

Link to publication

\section{General rights}

Copyright and moral rights for the publications made accessible in the public portal are retained by the authors and/or other copyright owners and it is a condition of accessing publications that users recognise and abide by the legal requirements associated with these rights.

- Users may download and print one copy of any publication from the public portal for the purpose of private study or research.

- You may not further distribute the material or use it for any profit-making activity or commercial gain

- You may freely distribute the URL identifying the publication in the public portal.

If the publication is distributed under the terms of Article 25fa of the Dutch Copyright Act, indicated by the "Taverne" license above, please follow below link for the End User Agreement:

www.tue.nl/taverne

Take down policy

If you believe that this document breaches copyright please contact us at:

openaccess@tue.nl

providing details and we will investigate your claim. 


\title{
Multivariable control design for fixed direction disturbances
}

\author{
Matthijs Boerlage, Bram de Jager, Maarten Steinbuch \\ Technische Universiteit Eindhoven \\ Dept. of Mechanical Engineering \\ P.O. Box 513, 5600 MB Eindhoven, The Netherlands \\ M.L.G.Boerlage@tue.nl, A.G.de.Jager@tue.nl, M.Steinbuch@tue.nl
}

\begin{abstract}
In this paper, a blind identification method is employed to model multivariable disturbances with fixed direction. The multivariable disturbance model is used to design nondiagonal weighting filters for $\mathcal{H}_{\infty}$ control. It is demonstrated that in this way, intuitive shaping of the directions of closed loop transfer functions is facilitated, maximally exploiting design freedom that has no analogue for scalar systems.
\end{abstract}

\section{INTRODUCTION}

Attenuation of disturbances is a key issue in control design. In multivariable systems, quite often, some physical causes (sources) generate disturbances on more controlled variables. The ratio in which these sources are dispersed over the controlled variables is the direction of the disturbance. In many applications, the fixed physical architecture of the plant means that the direction of these disturbances is fixed. The question then arises how directions of disturbances can be identified and how they can be used in multivariable control design.

In most cases, physical sources cannot be measured directly, only an unknown mixture of sources can be observed as disturbances entering at a certain point in the feedback loop. Identification of both the sources and the mixture, from a batch of observations boils down to a blind identification problem. A wide variety of methods has recently been developed to solve this problem in information theory, image processing and telecommunications, see [4] for a survey. Recently, it was demonstrated how these techniques can be used for diagnostics in industrial processes, [12]. As it is possible to recover the spatial structure of disturbance models, the question arises how this structure can be used in modeling multivariable disturbances.

For scalar systems, there exists an extensive line of research were specific properties of disturbances are identified and utilized to make meaningful control design tradeoffs, see, e.g., [1], [9]. Directions of disturbances, and their alignment to closed loop transfer functions play an important role in the achievable performance of multivariable systems, as was recognized in [7]. Hence, it is sensible to take directions of disturbances in account when shaping closed loop sensitivity functions, [6, p. 85]. In [5] an extensive study was undertaken on fundamental limitations for multivariable systems. It was shown that taking directions of disturbances into account facilitates use of design freedom that is unique for multivariable systems.

In this work, we propose a method to model fixed direction disturbances using techniques from blind identification. It is shown how these models lead straightforwardly to the choice of non-diagonal weighting filters for $\mathcal{H}_{\infty}$ control design. Hence, one is able to shape directions of closed loop transfer functions in a transparent way, exploiting design freedom mentioned above.

The paper is organized as follows. The next section shows how blind identification techniques can be used to model fixed direction disturbances. Section III discusses how these disturbance models can be used to design weighting filters. The theory is illustrated by means of a simulation example of a two dimensional manipulator in Section IV. The last section closes with conclusions and recommendations for future research.

\section{FIXED DIRECTION DISTURBANCES}

In this paper, we focus on the case depicted in Fig. 1. Herein, a controller $K$ is to be designed for the plant $G$ that suffers from disturbances $d$ at its outputs. The disturbances $d$ can be measured, both $G_{d}$ and $s$ are unknown. Often,

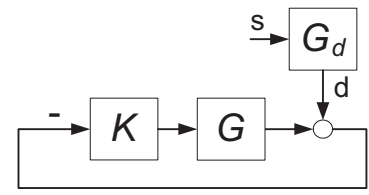

Fig. 1. Block diagram of controller architecture. The disturbance $d$ enters the loop at the output of the plant $G$. A controller $K$ is to be designed.

the location of a disturbance source is fixed so that the relation between the sources $s$ and the disturbances $d$ can be considered to be a constant transfer function, at least in the frequency region of interest. It is then justified to model disturbances as a constant mixture of physical sources,

$$
d(t)=G_{d} s(t)
$$

The columns of the matrix $G_{d}$ hold the directions of the disturbances. We assume that the sources $s(t)$ are mutually statistical independent. Sources are mutually statistical 
independent when their joint probability density function factorizes into the product of their marginal densities,

$$
p_{s_{1}, s_{2}, \ldots, s_{n}}\left(s_{1}, s_{2}, \ldots, s_{n}\right)=\prod_{i=1}^{n} p_{s_{i}} .
$$

This implies that knowing the value of one source, does not provide any information about the other sources. Hence by finding independent sources, one identifies the disturbances with minimum mutual information. Sources that are independent are also uncorrelated, but in general not vice versa. It is assumed that the disturbance sources are time colored, $R_{s}(\tau)=E\left\{s(t) s(t+\tau)^{H}\right\} \neq 0, \tau \geq 0$. As the disturbance sources originate from physical phenomena, each having dynamic behavior, this assumption is justified. Note that as the sources are independent, $R_{s}(\tau)$ is diagonal.

The objective is to recover the independent sources $s(t)$ from a batch of observations $d(t), t=\{0, \ldots, N\}$ without a-priory knowledge of $G_{d}$ and $s(t)$. This blind identification problem cam be solved using the Second Order Blind Identification (SOBI) method of [2]. Herein, the sources and the mixture can be retrieved, up to some indeterminacies in scaling and permutation. Later in this paper, it is shown that these indeterminacies are irrelevant for control design. For notational clarity we assume that the sources have zero mean. The SOBI method consists of two steps, namely 1) whitening and 2) joint diagonalization. These will be briefly discussed below.

\section{A. Whitening}

With this step, uncorrelated components $z(t)$ are recovered from the observed disturbances. The sources are then determined up to a unitary matrix $U$, so that

$$
d(t)=W z(t)=W U s(t)
$$

The covariance matrix of $d(t)$ at $\tau=0$ equals

$$
R_{d}(0)=W R_{z}(0) W^{T}=W U R_{s}(0) U^{T} W^{T} .
$$

Without loss of generality, we may assume that $R_{s}(0)=I$ so that

$$
R_{d}(0)=W W^{T}
$$

where $W$ is to be found. We use the singular value decomposition of the covariance of $d(t), R_{d}(0)=U_{d} \Sigma_{d} V_{d}^{H}$, [10]. As $R_{d}(0)$ is symmetric, we have $V_{d}=U_{d}$. So that,

$$
W=U_{d} \Sigma_{d}^{\frac{1}{2}}
$$

The directions of the disturbance are contained in the columns of $U_{d}$. The worst case disturbance has the same direction as the first column of $U_{d}$. By selecting only a few columns of $U_{d}$, one can reduce the number of modeled sources (less sources than channels) or eliminate noise with a specific structure [10]. In that case, $W$ is non-square. We still did not determine the sources uniquely as $R_{z}(0)$ is invariant for any unitary matrix $U$. Hence, we have recovered the sources up to this unknown unitary matrix,

$$
z(t)=U s(t) \text {. }
$$

\section{B. Joint diagonalization}

The matrix $U$ can be determined within some indeterminacies by using the fact that the sources are statistically independent. The covariance of $z(t)$ at $\tau \neq 0$ equals

$$
R_{z}(\tau)=U R_{s}(\tau) U^{T}
$$

where $R_{z}(\tau)$ is normal, symmetric and non-diagonal, $U$ is unitary and $R_{s}(\tau)$ is diagonal. Finding $U$ for a set of $\tau_{k}=\left\{\tau_{1}, \ldots, \tau_{N_{k}}\right\}$ is a unitary simultaneous diagonalization problem, [3], which can be solved within two indeterminacies; namely sign and permutation of the columns of $U$. We express these indeterminacies with the matrix $P$ which equals the product of a permutation matrix and a phase matrix. The solution $M P=U$, with unknown $P$, is the approximated eigenstructure of $R_{z}\left(\tau_{k}\right)$ for $\tau_{k}=\left\{\tau_{1}, \ldots, \tau_{N_{k}}\right\}$. This boils down to solving a minimization problem, e.g., using an extended Jacobi algorithm [3].

Combining the whitening and the joint diagonalization step, we find the following solution to the blind identification problem

$$
d(t)=W M P s(t)=\hat{G}_{d} \hat{s}(t)
$$

where $\hat{G}_{d}=W M$. It is clear that $P$ implies arbitrary ordering and arbitrary sign of the recovered sources. Note that the indeterminacies have no influence on the spectra. Hence, the spectra of the estimated sources equal the spectra of the true sources. In the whitening step, it was assumed that $R_{s}(0)=I$, which implies that all scalings of the sources are contained in $\hat{G}_{d}$. Alternatively, one may choose to scale the columns of $\hat{G}_{d}$ to unity. This is just a matter of convention.

\section{LOOPSHAPING FOR FIXED DIRECTION DISTURBANCES}

The objective is to find a stabilizing controller that minimizes the effects of the disturbances on the performance criteria. Fundamental limitations in achieving this, can be studied considering closed loop sensitivity functions. For linear time invariant multivariable systems, it was shown [5], that for a system with stable open loop transfer function $L(s)=G(s) K(s)$, the following integral constraint holds,

$$
\sum_{i} \int_{0}^{\infty} \ln \sigma_{i}\left(S_{o}(j \omega)\right) d \omega=0
$$

where $\sigma_{i}\left(S_{o}\right)$ is the $i^{t h}$ principal gain of the output sensitivity matrix, $S_{o}(j \omega)=(I+L(j \omega))^{-1}$. Each $i^{\text {th }}$ principal gain has a principal subspace $\mathcal{D}_{i}$. Due to this integral constraint, both frequency wise (waterbed effect) and spatial (between principal gains) tradeoffs of a multivariable sensitivity function are to be made. The spatial tradeoffs are typical for multivariable systems and imply that decreasing the sensitivity function in direction $\mathcal{D}_{i}$ increases the sensitivity function in orthogonal subspaces $\mathcal{D}_{j}, j \neq i$. Furthermore, algebraic tradeoffs hold as $S_{o}+T_{o}=I$, where $T_{o}=L(I+L)^{-1}$ is the output complementary sensitivity. In a real design case, all three design tradeoffs are to be handled simultaneously. Here, 
we focus on the rejection of output disturbances effecting the servo error. Therefore, we focus on minimizing the output sensitivity function in the frequency range and in the directions of interest. For illustration purposes, we weight all other closed loop functions uniformly, that is, we apply the same weight in all directions. We use loopshaping within the $\mathcal{H}_{\infty}$ framework, see, e.g., [8]. The control problem is to find a stabilizing controller $K$ so that, together with other objectives,

$$
\left\|S_{o} V\right\|_{\infty}
$$

is minimized. Herein, $V$ is a rational, stable, non-minimum phase, frequency dependent weighting filter that represents the frequencies and directions of the output disturbances. We consider three approaches to redesign $V$. Namely, designs with 1) disturbance direction fixed, sources unknown, $V_{d}$, 2) disturbance direction not fixed, hence worst case design, $\left.V_{w c}, 3\right)$ disturbance direction fixed and sources blindly identified, $V_{\hat{s}}$.

1) Disturbance direction fixed, sources unknown: Herein we assume that the direction of the disturbances is fixed, but the sources are not explicitly identified. We model directly the image of the disturbance model, hence structural information of the disturbance sources is not used. The disturbances at each plant output are modeled as if they have no mutual information. This is common practice in many decentralized control problems where controllers are independently designed for each output of a plant. A diagonal weighting filter is then chosen so that $V=V_{d}$,

$$
V_{d}(j \omega)=\operatorname{diag}\left\{V_{d, i}(j \omega)\right\}
$$

where $V_{d, i}(j \omega)$ is designed to satisfy

$$
\left|V_{d_{i}}(j \omega)\right| \geq\left|\sqrt{\Phi_{d_{i}}(j \omega)}\right|
$$

where $\Phi_{d_{i}}(j \omega)$ is the power spectrum of the output disturbance on each $i^{\text {th }}$ output of the plant.

2) Disturbance direction not fixed, hence worst case: When the direction is not fixed, a weighting filter must be designed to model a worst case disturbance in all directions. The worst case disturbance is the disturbance that results from the first whitened component (the first principal component, [4]). Using (6), we have that $d(t)=W z(t)$, so that the worst case disturbance equals;

$$
d_{w c}(t)=\tilde{w}_{1} z_{1}(t)
$$

where $\tilde{w}_{1}$ is the first column of $W$. We normalize the columns of $\tilde{w}_{1}$ to unity and move all scaling to the signal $z_{w c}(t)=\left\|\tilde{w}_{1}\right\|_{2} z_{1}(t), \tilde{w}_{w c}=\tilde{w}_{1}\left\|\tilde{w}_{1}\right\|_{2}^{-1}$. So that without loss of generality;

$$
d_{w c}(t)=\tilde{w}_{w c} z_{w c}(t)
$$

As it is not assumed that the disturbances have fixed direction, this weight is to be applied uniformly, that is, in all directions. The total weighting filter becomes $V=V_{w c}$,

$$
V_{w c}(j \omega)=V_{z, w c}(j \omega) I
$$

where $V_{z, w c}(j \omega)$ is a scalar weighting filter so that

$$
\left|V_{z, w c}(j \omega)\right| \geq\left|\sqrt{\Phi_{z_{w c}}(j \omega)}\right|
$$

is satisfied. Where $\Phi_{z_{w c}}(j \omega)$ is the power spectrum of $z_{w c}(t)$.

3) Disturbance direction fixed, blindly identified: Using the blind identification procedure from Section II, the independent components $\hat{s}(t)$ and the constant matrix $\hat{G}_{d}$ are recovered, so that the disturbance direction is fixed and known. We normalize the columns of $\hat{G}_{d}$ to unity. Hence, we define a diagonal scaling matrix $\Lambda=\operatorname{diag}\left\{\lambda_{i}\right\}$, where $\lambda_{i}=\left\|\hat{g}_{d, i}\right\|_{2}^{-1}$ is the inverse of the norm of the $i^{t h}$ column of $\hat{G}_{d}$. Now, $\hat{G}_{d}=\tilde{G}_{d} \Lambda$ and

$$
d(t)=\tilde{G}_{d} \tilde{s}(t)
$$

where $\tilde{s}(t)=\Lambda \hat{s}(t)$. Only $\tilde{G}_{d}$ contains the directions of each independent component whereas all magnitude information is held by $\tilde{s}(t)$. We define the total weighting filter $V=V_{\hat{s}}$, which is designed as

$$
V_{\hat{s}}(j \omega)=\tilde{G}_{d} V_{\tilde{s}}(j \omega)
$$

where $V_{\tilde{s}}(j \omega)$ is diagonal ( $V_{\hat{s}}(j \omega)$ is non diagonal) with on each $i^{\text {th }}$ diagonal element a weighting filter for the power spectrum of the $i^{\text {th }}$ (scaled) independent component.

$$
\left|V_{\tilde{s}_{i}}(j \omega)\right| \geq\left|\sqrt{\Phi_{\tilde{s}_{i}}(j \omega)}\right| .
$$

The indeterminacies of the blind identification solution only imply the exchange of gain, sign and permutation between $\hat{s}(t)$ and the columns of $\hat{G}_{d}$, hence these have no nett effect on $V_{\hat{s}}(j \omega)$.

\section{Simulation Example}

In this section, the design of weighting filters for disturbances is illustrated on a model of a two degrees of freedom manipulator. The plant is diagonal, so that the plant can be controlled independently in each direction. We choose a diagonal plant for clarity, the theory is certainly not limited to this,

$$
G(s)=\left[\begin{array}{cc}
\frac{k_{1}}{s^{2}} & 0 \\
0 & \frac{k_{2}}{s^{2}}
\end{array}\right] .
$$

Here, $k_{1}=k_{2}=0.25 \times 10^{-3}$, which results in a magnitude of $0 \mathrm{~dB}$ at $10 \mathrm{~Hz}$ for each controlled axis. The plant dynamics are uncertain at frequencies above $100[\mathrm{~Hz}]$. The output disturbances are shown in Fig.2. After identification of the sources, various control designs are illustrated.

\section{A. Blind identification}

Using the observed output disturbances, the whitening procedure is performed to find the uncorrelated components $z(t), d(t)=W z(t)$. This results in the following whitening matrix;

$$
W=\left[\begin{array}{cc}
-4.21 & -0.35 \\
-3.57 & 0.41
\end{array}\right]
$$


The uncorrelated components are shown in Fig. 2. It is clear that these uncorrelated components do not allow straightforward physical interpretation. Using joint diagonalization, the independent components $\hat{s}(t)$ are recovered

$$
\hat{s}(t)=\hat{G}_{d}^{-1} d(t)=(W M)^{-1} d(t)
$$

where $\hat{G}_{d}$ is determined as

$$
\hat{G}_{d}=\left[\begin{array}{ll}
-3.01 & -2.97 \\
-3.01 & -1.97
\end{array}\right] .
$$

For this example, the true (but unknown) matrix was

$$
G_{d}=\left[\begin{array}{ll}
3 & 3 \\
3 & 2
\end{array}\right]
$$

so that the $P$ in this example resulted in a change of sign of the recovered mixing matrix (and the same change of sign in the recovered sources). The independent components are shown in Fig. 2. Clearly, a step sequence and a combination
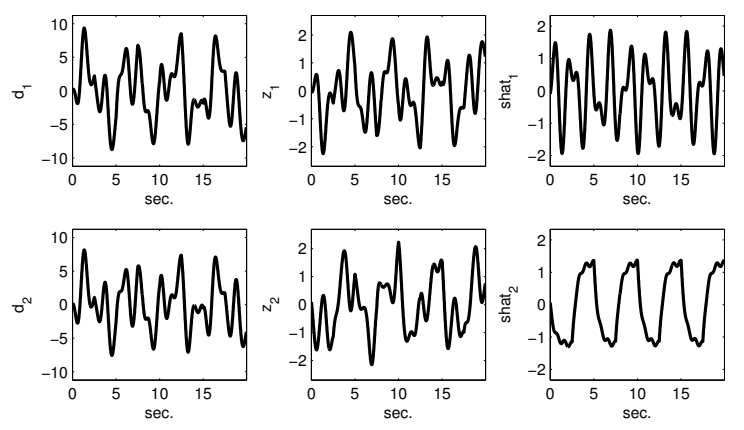

Fig. 2. Left column: output disturbances $d(t)$, Center column: uncorrelated components $z(t)$, Right column: independent components $\hat{s}(t)$

of harmonics can be distinguished. In a practical situation, one may interpret this as sources from physical phenomena (other machines in a factory, pumps, etc.).

\section{B. Control design}

With the identified sources, several controller designs are initiated. As the plant has uncertain dynamics above $100 \mathrm{~Hz}$, roll-off is desired. This requirement is expressed by weighting the control sensitivity $K S_{o}$ with $W_{k s}$. Also, we have a low frequency objective, expressed by a weight $W_{s}$ on the output sensitivity. These two objectives are stacked in a standard $\mathcal{H}_{\infty}$ mixed sensitivity problem, see, e.g., [8]. Now a stabilizing controller $K$ is to be found which minimizes

$$
\left\|\begin{array}{c}
W_{s} S_{o} V \\
W_{k s} K S_{o} V
\end{array}\right\|_{\infty} .
$$

The weighting filters $W_{s}, W_{k s}$ are chosen diagonal, with elements whose frequency response is shown in Fig. 3. These weighting filters are the same for all examples. The initial design is $V=I$. Choosing a particular $V$, the relevant frequencies and directions of the disturbances can be taken into account. Alternative choices of $V$ are studied, each using a different disturbance model. All designs are

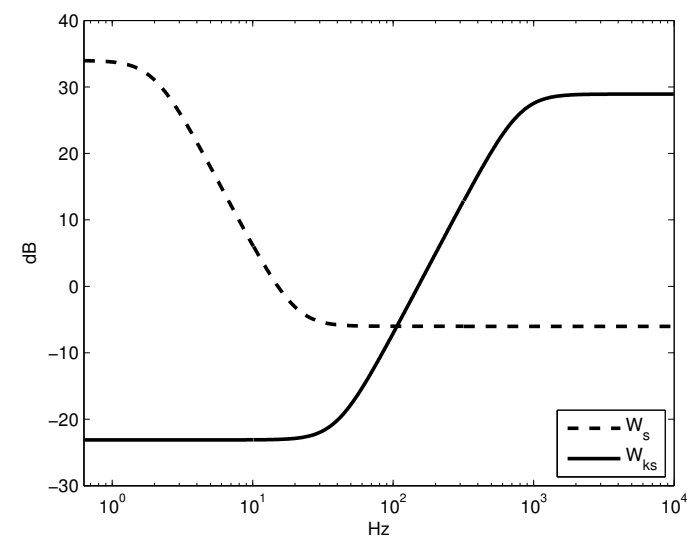

Fig. 3. Frequency response (magnitude only) of the elements of the weighting filters (diagonal) for the sensitivity function $W_{s}$ (dashed) and the control sensitivity function $W_{k s}$ (solid).

targeted to achieve the same closed loop bandwidth with the same robustness margins.

Firstly, a design is discussed with a weighting filter that bounds the power spectra of $d(t), V=V_{d}$. Secondly, a weighting filter that bounds the worst case disturbance in all directions is presented, $V=V_{w c}$. Herein, it is assumed that the disturbance direction is not fixed. Finally, the design with a weighting filter is shown that uses the structure of the blindly identified independent components $\hat{s}(t), V=V_{\hat{s}}$. In all three designs, the power spectra are bounded above with second order weighting filters. Hence, each weight filter $V$ is of fourth order. Furthermore, each weighting filter $V$ is designed to be equal to $I$ at frequencies close to the bandwidth so that the margins and bandwidth of all designs are comparable. This is done just for illustration purposes and is no limitation in the theory presented here.

1) Design $V_{d}(j \omega)$ : The power spectra of $d(t)$ are calculated. Each spectrum contains contributions of both disturbance sources. A second order weighting filter is designed to bound each spectrum from above, Fig. 4.

2) Design $V_{w c}(j \omega)$ : The power spectrum of the worst case disturbance (the first principal component) is calculated. The worst case disturbance contains contributions of both sources. A second order weighting filter is used to bound the power spectrum from above, Fig. 5. This weighting is applied in all directions.

3) Design $V_{\hat{s}}(j \omega)$ : The power spectra of $\tilde{s}(t)(\hat{s}(t)$ scaled with $\Lambda$ ) are calculated. As the sources are independent, the spectra are distinct. A second order low pass weighting filter bounds the power spectrum of each source from above, Fig. 6. The total weighting filter must be equal to the identity matrix at frequencies around the bandwidth, hence, we choose;

$$
V_{\hat{s}}(j \omega)=\tilde{G}_{d} V_{\tilde{s}}(j \omega)-I .
$$




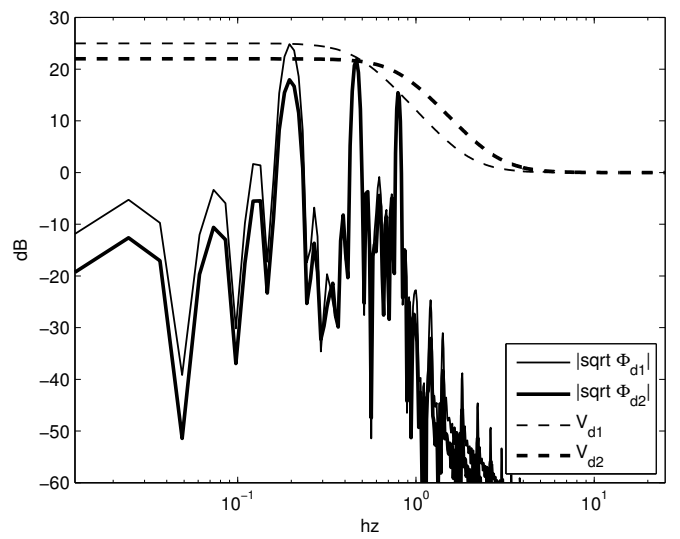

Fig. 4. Weighting filter design based on the power spectrum of the output disturbances.

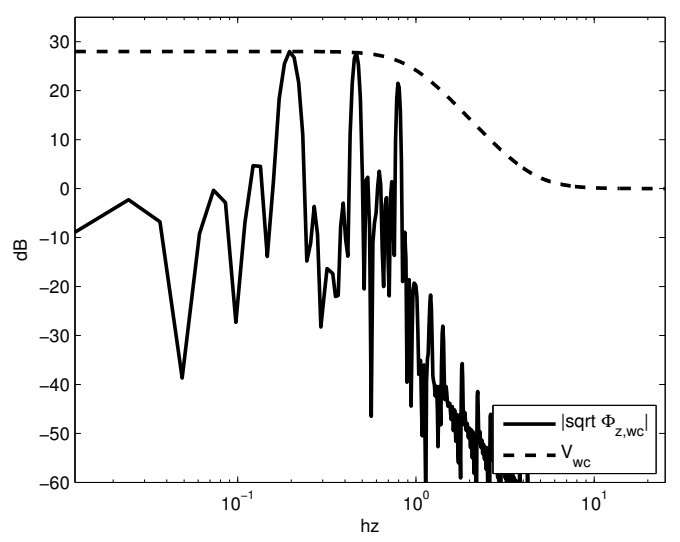

Fig. 5. Weighting filter design based on the power spectrum of the worst case disturbance.

Herein, $V_{\tilde{s}}$ is diagonal and $V_{\hat{s}}$ is non-diagonal.

In all three cases the weighting filters have limited order, hence conservatism is introduced in bounding the power spectra. This conservatism increases significantly when the power spectra contain contributions of more sources, as in the case of $V_{d}, V_{w c}$. In the design of $V_{\hat{s}}$, the spectra are more distinct. Hence, one may reduce conservatism. In addition, the choice of the weighting filters can be related to each independent physical cause. The bode magnitude plot of all three weighting filters is depicted in Fig. 7. It is clear that both $V_{w c}$ and $V_{d}$ are diagonal, $V_{\hat{s}}$ is non-diagonal.

The principal gains of each weighting filter are depicted in Fig. 8. Both $V_{d}$ and $V_{w c}$ have high gains in all principal directions. Hence, weights are applied in directions that are not reached by the disturbances. The weighting filter $V_{\hat{s}}$ weights only in the relevant directions and at the same time, reduces weight in orthogonal directions. As a result, the design with $V_{\hat{s}}$ allows the sensitivity to increase in directions orthogonal to the disturbance directions, Fig. 9.

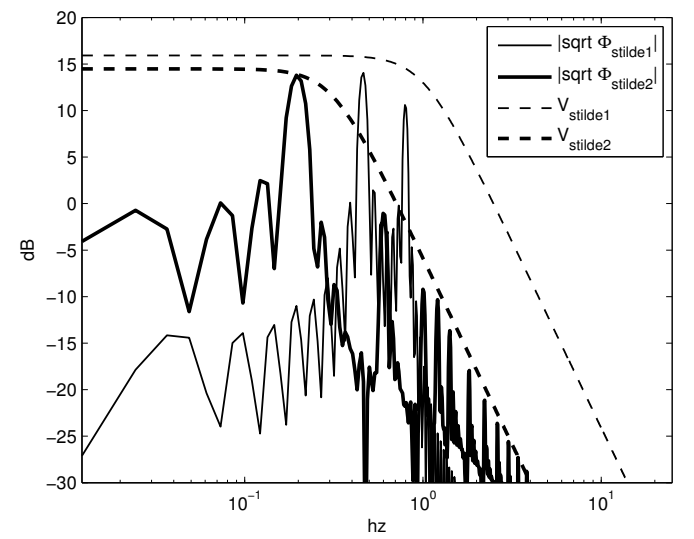

Fig. 6. Weighting filter design based on the power spectrum of the independent components.
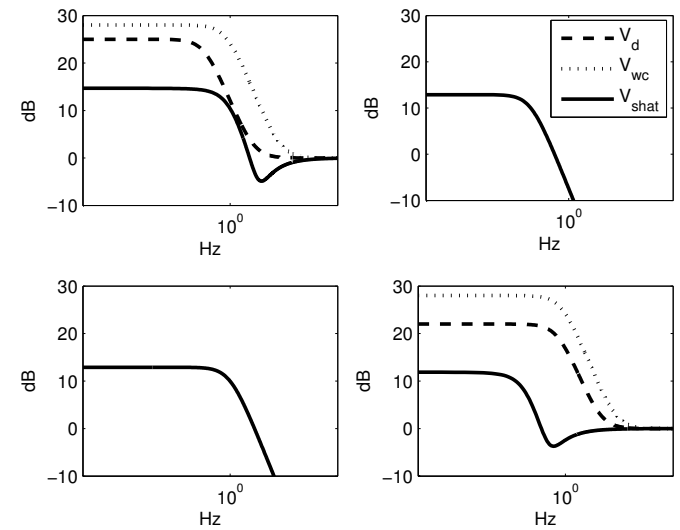

Fig. 7. Bode magnitude plot of all three weighting filters, $V_{d}$ (dashed), $V_{w c}$ (dotted), $V_{\hat{s}}$ (solid)

In this case, the nett effect of the weight $V_{\hat{s}}$ is equal to the initial weight $V=I$.

\section{Multivariable design freedom}

In order to illustrate the use of multivariable design freedom, the integral constraint in (10) is studied. The singular values of the output sensitivities are calculated over a linear frequency grid $f=\left[\begin{array}{ll}0.01 & 1 e 3\end{array}\right][h z]$ with $1 \times 10^{4}$ points. Then, the surface is calculated between each principal gain and $0 d B$ for the intervals where the principal gains are below and above $0 d B$ respectively, see Table I. Here, $O_{\sigma_{i}<1}$ denotes the surface between $\sigma_{i}\left(S_{o}\right)$, and $0 d B$ when $\sigma_{i}\left(S_{o}\right)$ is below $0 d B, O_{\sigma_{i}>1}$ denotes above. The total surface between $\sigma_{i}\left(S_{o}\right)$ and $0 d B$ when $\sigma_{i}\left(S_{o}\right)$ is above $0 d B$, is denoted as $O_{s u m \sigma_{i}}$. Both the design with $V_{w c}$ and $V_{d}$ show a significant increase of the sensitivity function above $0 d B$, were the design with $V_{\hat{s}}$ has almost the same costs at high frequencies as the initial design with $V=I$. Hence, the design with $V_{\hat{s}}$, which attenuates disturbances in one direction, does not entail costs by means of the waterbed effect. 


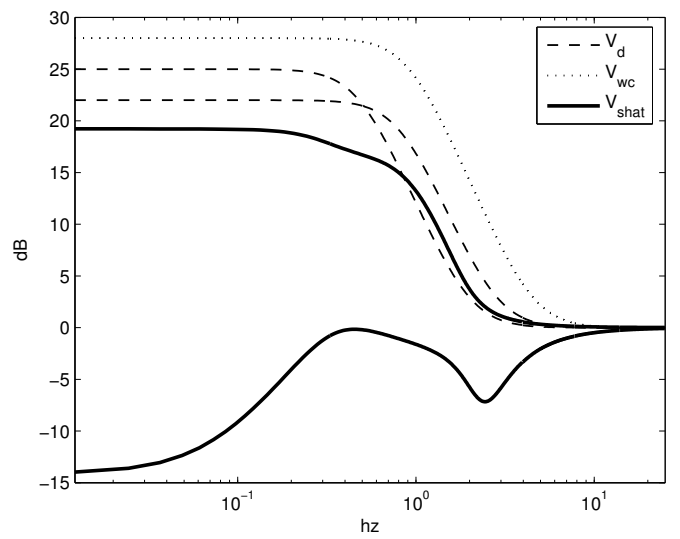

Fig. 8. Principal gains of the weighting filters $V_{d}$ (dashed), $V_{w c}$ (dotted) and $V_{\hat{s}}$ (solid).
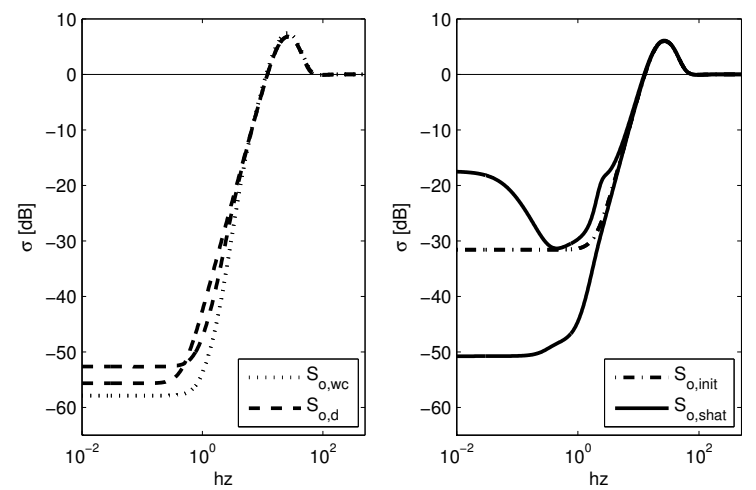

Fig. 9. Principal gains of output sensitivities with four choices of $V$. Left: design with weight filter based on the output disturbances (dashed) and design for worst case disturbance in all directions (dotted). Right: initial design with $V=I$ (dash dot) and design based on the independent components (solid).

In the design with $V_{\hat{s}}$, the weighting of the sensitivity function in the direction of the disturbances is compensated for by reducing weight on the sensitivity function in directions orthogonal to the disturbances, Fig. 9. Hence, the sum of the surface between the principal gains and $0 d B$ of $V_{\hat{s}}$ is close to zero and there is no nett effect on the integral constraints. Therefore, sensitivity is traded off spatially instead of frequency wise, so that the well known waterbed effect, [1] is avoided. Hence, it is demonstrated that by taking into account the directions of the disturbances, design freedom can be exploited that has no scalar analogue.

\section{CONCLUSion}

In this work, we employed a method to identify independent sources and their directions from observations of output disturbances. It was shown that using this knowledge, transparent design of weighting filters is facilitated. The spectra of independent sources are distinct, leading to less conservative choice of weighting filters and
TABLE I

Cumulative Sum of THE SURFACES Below $\ln \sigma_{i}\left(S_{o}\right)$

\begin{tabular}{|c||c|c|c|c|c|}
\hline & $O_{\sigma_{1}<1}$ & $O_{\sigma_{2}<1}$ & $O_{\sigma_{1}>1}$ & $O_{\sigma_{2}>1}$ & $O_{\text {sum } \sigma_{i}>1}$ \\
\hline \hline$V=I$ & -212 & -212 & 210 & 210 & 421 \\
\hline \hline$V=V_{d}$ & -232 & -251 & 243 & 236 & 479 \\
\hline \hline$V=V_{w c}$ & -271 & -271 & 268 & 268 & 536 \\
\hline \hline$V=V_{\hat{s}}$ & -181 & -246 & 214 & 210 & 425 \\
\hline
\end{tabular}

potentially lower order controllers. Also, as the independent sources are strongly related to the true physical phenomena, physical interpretation of the disturbances is facilitated. As the structure of the sources can be taken into account, only relevant directions are weighted and conservatism reduces significantly. This allows the sensitivity function to increase in directions where disturbances do not occur. Therefore, performance can be traded off both spatially and frequency wise. Hence, multivariable design freedom can be exploited that has no scalar analogue.

The method proposed in this work can straightforwardly be extended to handle exogenous signals other than output disturbances. Furthermore, control design can be carried out for each source at a time, so that complexity of a multivariable control system can be gradually increased. We focussed on disturbances whose direction does not change with frequency. More general blind identification techniques, as in [11], are currently investigated in order to increase the application area.

\section{REFERENCES}

[1] D. Abramovitch, T. Hurst, and D. Henze, "The PES Pareto method: Uncovering the strata of position error signals in disk drives," in Proceedings of the American Control Conference, 1997.

[2] A. Belouchrani, K. Abed-Meraim, J. Cardoso, and E. Moulines, "A blind source separation technique using second-order statistics," IEEE Transactions on Signal Processing, vol. 45, no. 2, pp. 123-145, 1997.

[3] J. Cardoso and A. Soulmiac, "Jacobi-angles for simulteneous diagonalization," SIAM J. Mat. Anal. Appl., vol. 17, no. 1, pp. 161-164, 1996.

[4] P. Comon, "Independent component analysis, a new concept?" Signal Processing, vol. 36, no. 3, pp. 287-314, 1994.

[5] J. Freudenberg and D. Looze, Frequency Domain Properties of Scalar and Multivariable Feedback Systems, Lecture Notes in Control and Information Sciences, M. Thoma and A. Wyner, Eds. SpringerVerlag, 1988.

[6] J. Maciejowski, Multivariable feedback design. Addison-Wesley, 1989.

[7] S. Skogestad and M. Morari, "Effect of disturbance directions on closed-loop performance," Ind. Eng. Chem. Res., vol. 26, no. 1, pp. 2029-2035, 1987.

[8] S. Skogestad and I. Postlethwaite, Multivariable feedback control. Wiley, 2005.

[9] M. Steinbuch and M. Norg, "Advanced motion control: an industrial perspective," European J. of Control, vol. 4, no. 1, pp. 278-293, 1998.

[10] L. Tong, V. Soon, R. Liu, and Y. Huang, "AMUSE a new blind identification algorithm," in Proceedings of the ISCAS, 1990.

[11] J. Tugnait, "Identification and deconvolution of multichannel linear non-Gaussian processes using higher order statistics and inverse filter criteria," IEEE Transaction on Signal Processing, vol. 45, no. 3, pp. 658-672, 1997.

[12] C. Xia and J. Howell, "Isolating multiple sources of plant-wide oscillations via independent component analysis," Control Engineering Practice, vol. 13, no. 8, pp. 1027-1035, 2005. 